\title{
L'impact du statut vaccinal sur l'importation de la COVID-19 chez les voyageurs internationaux
}

\author{
Paul Ronksley*, Tayler Scory², Robert Weaver ${ }^{2}$, Meaghan Lunney ${ }^{1}$, Rachel Rodin ${ }^{3}$, Marcello Tonelli²
}

\section{Résumé}

Les gouvernements du monde entier cherchent des moyens de permettre les voyages internationaux en toute sécurité tout en atténuant la propagation du coronavirus du syndrome respiratoire aigu sévère 2 (SRAS-CoV-2) et de la maladie à coronavirus 2019 (COVID-19) qui lui est associée. Cependant, peu de données décrivent l'impact de la vaccination sur I'importation de la COVID-19. Nous avons profité de l'introduction séquentielle de deux politiques gouvernementales au Canada pour évaluer les preuves réelles de l'efficacité du vaccin chez 30361 voyageurs internationaux arrivant par avion en Alberta, au Canada. La proportion de résultats positifs au test de dépistage de COVID-19 chez les voyageurs vaccinés ou partiellement vaccinés était de $0,02 \%(95 \%$ IC : 0,00-0,10) (soit un cas positif sur 5817 voyageurs). En revanche, 1,42\% (95\% IC : 1,27-1,58) des voyageurs non vaccinés ont reçu un résultat de test positif pour le SRAS-CoV-2 (341 cas parmi 24034 voyageurs). Ces résultats suggèrent que les vaccins contre la COVID-19 approuvés au Canada, ont considérablement réduit le risque d'importation de la COVID-19 lié aux voyages lorsqu'ils sont associés à d'autres mesures de santé publique. Le faible taux absolu d'infection chez les voyageurs internationaux vaccinés ou partiellement vaccinés peut éclairer les exigences de quarantaine dans cette population.
Cette oeuvre est mise à la disposition selon les termes de la licence internationale Creative Commons Attribution 4.0

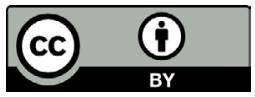

\begin{abstract}
Affiliations
${ }^{1}$ Département des sciences de la santé communautaire, École de médecine Cumming, Université de Calgary, Calgary, AB

2 Département de médecine, École de médecine Cumming, Université de Calgary, Calgary, AB

${ }^{3}$ Bureau de la vice-présidente, Agence de la santé publique du Canada, Ottawa, ON
\end{abstract}

\section{*Correspondance :}

peronksl@ucalgary.ca

Citation proposée : Ronksley PE, Scory TD, Weaver RG, Lunney M, Rodin R, Tonelli M. L'impact du statut vaccinal sur l'importation de la COVID-19 chez les voyageurs internationaux. Relevé des maladies transmissibles au Canada 2021;47(11):525-7. https://doi.org/10.14745/ccdr.v47i11a05f

Mots-clés : COVID-19, vaccination, voyages internationaux, importation de cas

\section{Introduction}

Les gouvernements du monde entier cherchent des moyens de permettre les voyages internationaux en toute sécurité tout en atténuant la propagation du coronavirus du syndrome respiratoire aigu sévère 2 (SRAS-CoV-2) et de la maladie à coronavirus 2019 (COVID-19) qui lui est associée. Les campagnes de vaccination sont en cours dans de nombreux pays à revenu élevé et des données probantes indiquant que les personnes vaccinées sont moins susceptibles d'être infectées s'accumulent. Peu de données décrivent l'impact de la vaccination sur l'importation de la COVID-19, un indicateur potentiellement utile pour contribuer à la politique frontalière. En utilisant les données de deux programmes de dépistage aux frontières parrainés par le gouvernement, nous fournissons des preuves concrètes de l'efficacité du vaccin chez 30361 voyageurs internationaux arrivant par avion à l'aéroport international de Calgary.

\section{Situation actuelle}

En mars 2020, le gouvernement fédéral canadien a fermé les frontières à tout le monde, sauf aux personnes répondant à certains critères (1). Parmi les personnes autorisées à franchir la frontière, un groupe était appelé "voyageurs exemptés ", et comprenait les membres des équipages de vol, ceux qui transportent des marchandises par la frontière canadoaméricaine, et d'autres qui fournissent certains services essentiels. Les autres personnes autorisées à franchir la frontière étaient appelées "voyageurs non exemptés ", notamment les citoyens canadiens, les résidents permanents, les membres de la famille immédiate des citoyens/résidents permanents et d'autres personnes ayant une raison précise de voyager, comme la réunification familiale. L'aéroport international de Calgary était l'un des quatre aéroports qui restaient ouverts aux vols en provenance de l'extérieur du Canada, des États-Unis et des Caraïbes, avec les aéroports de Montréal, Toronto et Vancouver. 
Les données de la période du 6 janvier 2021 au 21 février 2021 ont été obtenues auprès de voyageurs non exemptés qui avaient le droit d'entrer au Canada, qui sont arrivés par avion et qui ont participé au Programme pilote de dépistage de la COVID-19 aux frontières de l'Alberta (2). Le Programme pilote de dépistage de la COVID-19 aux frontières de l'Alberta a été suspendu à 23 h 59 HNE le 21 février 2021 et tous les voyageurs internationaux arrivant en Alberta par voie aérienne par la suite ont été soumis à une procédure d'entrée obligatoire à la frontière (3). Nous présentons ici un rapport sur les voyageurs du Programmepilote de dépistage de la COVID-19 aux frontières de l'Alberta et du programme obligatoire du 6 janvier 2021 au 23 mai 2021, appelés ci-après les « participants ».

Tous les voyageurs visés par le présent rapport devaient présenter la preuve d'un test moléculaire négatif pour le SRAS-CoV-2 (e.g. amplification en chaîne par polymérase (PCR), test des acides nucléiques (TAN), amplification isotherme induite par boucle de transcription inverse (RT-LAMP), etc.), effectué moins de 72 heures avant le départ prévu pour le Canada (4), et subir un autre test moléculaire 7 à 8 jours après leur arrivée. Le statut de vaccination complète était défini par la réception autodéclarée de deux doses d'un vaccin contre la COVID-19 approuvé au Canada (5) au moins 14 jours avant la date d'arrivée. La vaccination partielle était définie par la réception autodéclarée d'une dose de vaccin contre la COVID-19 approuvé par le Canada avant la date d'arrivée ou de deux doses de vaccin contre la COVID-19 approuvé par le Canada moins de 14 jours avant la date d'arrivée. Les personnes ayant reçu une seule dose de vaccin ont été considérées comme partiellement vaccinées. Les personnes n'ayant pas déclaré leur statut vaccinal ont été liées, dans la mesure du possible, au registre provincial de vaccination afin de déterminer leur statut au moment de la date d'arrivée. La plupart des voyageurs internationaux étaient des résidents de l'Alberta revenant de l'étranger et ont fourni un numéro d'assurance-maladie provincial.
Parmi les 30361 voyageurs non exemptés, la majorité a voyagé sur des vols en provenance des États-Unis $(53,20 \%)$ et du Mexique (20,79\%). Leur âge médian était de 45,0 ans et 52,5\% étaient des hommes. 28658 (94,39\%) étaient âgés d'au moins 12 ans, dont 1595 (5,57 \%) étaient partiellement vaccinés et 4227 (14,75\%) étaient complètement vaccinés. La proportion de résultats positifs pour les participants qui étaient soit totalement vaccinés, soit partiellement vaccinés, était de $0,02 \%$ (95\% IC : 0,00-0,10 [i.e. un cas positif parmi les 5817 participants qui ont été testés pour la COVID-19] (tableau 1). En revanche, 1,42 \% (95 \% IC : 1,27-1,58) des participants non vaccinés ont reçu un résultat de test positif pour le SRAS-CoV-2 (341 cas parmi 24034 participants). Cela équivaut à un risque relatif de test positif chez les participants vaccinés ou partiellement vaccinés de 0,01 (95\% IC : 0,00-0,09) par rapport aux participants non vaccinés.

Le test positif chez le voyageur vacciné a été suivi d'un séquençage et s'est révélé négatif pour un variant préoccupant. Un autre échantillon a été obtenu de ce voyageur trois jours plus tard et un test moléculaire répété s'est révélé négatif pour le SRAS-CoV-2-ce qui a soulevé la possibilité d'un test initial faussement positif.

\section{Conclusion}

Ces résultats suggèrent que les vaccins contre la COVID-19 approuvés au Canada réduisent considérablement le risque d'importation de la COVID-19 lié aux voyages lorsqu'ils sont associés à d'autres mesures de santé publique. Le faible taux absolu d'infection chez les voyageurs vaccinés ou partiellement vaccinés non exemptés peut éclairer les exigences de quarantaine dans cette population. Cependant, compte tenu de la période couverte par le présent rapport, les travaux futurs devraient chercher à savoir si l'efficacité des vaccins pour atténuer l'importation de cas parmi les voyageurs internationaux a changé suite à l'augmentation récente des variants préoccupants (en particulier le variant Delta).

\section{Tableau 1 : Résultats du test de dépistage de la COVID-19 chez les voyageurs internationaux non exemptés arrivant en Alberta par avion entre le 6 janvier 2021 et le 23 mai 2021}

\begin{tabular}{|c|c|c|c|c|c|c|c|c|c|c|c|c|c|c|c|}
\hline \multirow{2}{*}{$\begin{array}{l}\text { Résultats } \\
\text { des tests }\end{array}$} & \multicolumn{3}{|c|}{ Vacciné } & \multicolumn{3}{|c|}{ Partiellement vacciné } & \multicolumn{3}{|c|}{ Non vacciné } & \multicolumn{3}{|c|}{ Manquant/inconnu } & \multicolumn{3}{|c|}{ Total } \\
\hline & $\mathbf{n}$ & $\%$ & $95 \%$ IC & $\mathbf{n}$ & $\%$ & $95 \%$ IC & $\mathbf{n}$ & $\%$ & $95 \%$ IC & $\mathbf{n}$ & $\%$ & $95 \%$ IC & $\mathbf{n}$ & $\%$ & $95 \%$ IC \\
\hline Positifa & 1 & 0,02 & $0,00-0,13$ & 0 & 0,00 & $0,00-0,23$ & 341 & 1,42 & $1,27-1,58$ & 0 & 0,00 & $0,00-7,11$ & 342 & 1,14 & $1,03-1,27$ \\
\hline Négatif & 4224 & 99,98 & - & 1592 & 100 & - & 23693 & 98,58 & - & 50 & 100 & - & 29559 & 98,85 & - \\
\hline $\begin{array}{l}\text { Pas de } \\
\text { test }\end{array}$ & 2 & - & - & 3 & - & - & 455 & - & - & 0 & - & - & 460 & - & - \\
\hline Total & 4227 & 13,92 & - & 1595 & 5,25 & - & 24489 & 80,66 & - & 50 & 0,16 & - & 30361 & 100 & - \\
\hline
\end{tabular}

Abréviations : IC, intervalle de confiance, -, non rapporté

a La proportion de résultats positifs pour les participants qui étaient vaccinés ou partiellement vaccinés était de 0,02\% (0,00\%, 0,10\%) 


\section{Déclaration des auteurs}

P. E. R. - Contributions à la conception et au design, interprétation des données; rédaction de la première ébauche R. G. W. - Contributions à la conception et au design, interprétation des données

M. L. - Contributions à la conception et au design, interprétation des données

R. R. - Contributions à la conception et au design, interprétation des données

M. T. - Contribution à la conception et au design, interprétation des données, rédaction de la première ébauche

T. D. S. - Analyse des données

Tous les auteurs ont participé à la révision critique du contenu intellectuel important. Tous les auteurs ont donné leur approbation finale à la version à publier et ont accepté d'être responsables de tous les aspects du travail.

\section{Intérêts concurrents}

Aucun.

\section{Financement}

Ce travail a été financé par un contrat du gouvernement de l'Alberta, qui a reçu une subvention de l'Agence de santé publique du Canada.

\section{Références}

1. Agence de la santé publique du Canada. Liste des lois et règlements. Ottawa (ON) : ASPC; 2021 (accédé 2021-06-07). https://www.canada.ca/fr/sante-publique/organisation/ mandat/a-propos-agence/lois-reglements/liste-loisreglements.html

2. Agence de la santé publique du Canada. Programme pilote de dépistage de la COVID-19 aux frontières de l'Alberta. Ottawa (ON) : Gouvernement du Canada; 2021 (accédé 2021-06-07). https://www.canada.ca/fr/sante-publique/ services/maladies/2019-nouveau-coronavirus/derniersconseils-sante-voyageurs/programme-pilote-depistagecovid-19-frontieres-alberta.html

3. Agence de la santé publique du Canada. Le gouvernement du Canada adopte des restrictions supplémentaires s'appliquant aux voyages internationaux. Ottawa (ON) : Gouvernement du Canada; 2021 (accédé 2021-06-07). https://www.canada.ca/fr/transports-canada/ nouvelles/2021/01/le-gouvernement-du-canada-adoptedes-restrictions-supplementaires-sappliquant-aux-voyagesinternationaux.html

4. Agence de la santé publique du Canada. Dépistage de la COVID-19 pour les voyageurs. Ottawa (ON) : Gouvernement du Canada; 2021 (accédé 2021-06-07). https://voyage. gc.ca/voyage-covid/voyage-restrictions/liste-verificationavion-canada/tests-depistage-covid-19-voyageurs-arriventcanada?_ga=2.254395855.654799052.1634748781 2039673885.1634748780

5. Agence de la santé publique du Canada. Vaccins approuvés contre la COVID-19. Ottawa (ON) : Gouvernement du Canada; 2021 (accédé 2021-06-07). https://www.canada. $\mathrm{ca} / \mathrm{fr} /$ sante-canada/services/medicaments-produits-sante/ covid19-industrie/medicaments-vaccins-traitements/vaccins. html 